\title{
Hyperubiquitylation of wild-type p53 contributes to cytoplasmic sequestration in neuroblastoma
}

\author{
K Becker ${ }^{1}$, ND Marchenko ${ }^{1}$, M Maurice ${ }^{2}$ and UM Moll ${ }^{*, 1}$
}

\begin{abstract}
Neuroblastoma (NB) is the most common solid malignancy in childhood and its prognosis is still generally poor. In contrast to many other cancers, mutations of the p53 tumor suppressor are rare. Instead, significant cytosolic sequestration of wtp53 is one of several mechanisms that attenuate p53 function in this cancer. Here, we report that aberrant p53 hyperubiquitylation contributes to p53 cytoplasmic sequestration in NB. NB lines constitutively harbor an elevated portion of wtp53 as stable ubiquitylated species confined to the cytoplasm. p53 hyperubiquitylation is not due to dysregulation by Hdm2 or proteasomal dysfunction. Instead, the defect lies in p53 regulation by HAUSP, a major p53-deubiquitylating enzyme. In contrast to non-NB cancer cells with nuclear p53 and normal ubiquitylation, p53 from NB cells shows impaired HAUSP interaction. Conversely, interference with p53 hyperubiquitylation in NB cells by Nutlin 3a or by a C-terminal p53 peptide (aa 305-393) results in p53 relocalization from the cytoplasm to the nucleus, and in case of Nutlin, in reactivation of p53's transcriptional and apoptotic functions. Moreover, nutlin and camptothecin act synergistically in inducing NB cell apoptosis. Hence, this study strengthens the rationale for targeting p53 deubiquitylation by drugs like Nutlin as a promising new strategy in NB therapy.
\end{abstract}

Cell Death and Differentiation (2007) 14, 1350-1360; doi:10.1038/sj.cdd.4402126; published online 23 March 2007

Neuroblastoma (NB) is the most common solid malignancy in childhood and still accounts for more pediatric cancer deaths than any other cancer type. ${ }^{1}$ The p53 tumor suppressor induces cell growth arrest, apoptosis and senescence in response to various types of stress and is mutated in over $50 \%$ of cancers. $^{2}$ However, in NB p53 mutations are exceedingly rare and occur in $<2 \%$, typically in relapsed tumors after therapy. ${ }^{3,4}$ Instead, subcellular mislocalization of wtp53 with constitutive cytoplasmic sequestration represents one of several alternative mechanisms to inactivate p53 function in this cancer. Moll et al. ${ }^{5}$ originally found cytoplasmic accumulation of p53 in $96 \%$ of primary undifferentiated NB tumors, whereas differentiated tumors (ganglioneuromas) lacked detectable levels of p53. Although some NB lines show predominantly nuclear $\mathrm{p} 53$ staining, ${ }^{6}$ many NB cell lines also exhibit abnormal cytoplasmic p53 localization. ${ }^{7-13}$ Cytoplasmic sequestration of wtp53 correlates with attenuation of DNA damage-induced $\mathrm{G} 1$ arrest ${ }^{6,7}$ and apoptosis ${ }^{13,14}$ in NB cell lines, since this interferes with p53's function as a transcription factor in the nucleus. Furthermore, Wolff et al. ${ }^{15}$ reported that wtp53 in NB is in a conformation that is refractory to integration into transcriptional complexes, adding to transcriptional inactivity.

In growing, unstressed cells p53 levels are very low owing to rapid degradation via the ubiquitin-proteasome pathway. Hdm2, the major E3 ligase which itself is a target gene of p53, ubiquitylates $\mathrm{p} 53$, thus constituting a negative-feedback loop. ${ }^{16,17}$ Importantly, this ubiquitylation of p53 is required for its nuclear export via the export receptor CRM 1. ${ }^{18-20}$ Furthermore, the fate of p53 depends on the type of ubiquitylation. Although polyubiquitylated p53 is unstable due to rapid proteasomal degradation, (multi)monoubiquitylated p53 is stable and results in nuclear exclusion. ${ }^{21}$ p53 ubiquitylation is reversible by the p53 deubiquitylating enzyme HAUSP (herpes virus-associated ubiquitin-specific protease), resulting in p53 stabilization, induction of growth arrest and apoptosis. ${ }^{22}$ The stability of $\mathrm{Hdm} 2$ (and the related protein $\mathrm{MdmX/4)}$ is also regulated by HAUSP. Thus, HAUSP targets both p53 and Hdm2 as substrates and in concert with $\mathrm{Hdm} 2$ plays a dynamic role in $\mathrm{p} 53$ regulation. ${ }^{23-25}$

Here, we report that in NB aberrant hyperubiquitylation of endogenous wild-type p53 owing to impaired p53-HAUSP interaction contributes to enhanced cytoplasmic sequestration and attenuation of p53 function. Moreover, HAUSP downregulation in non-NB cells cause a phenotype that also results in cytoplasmic accumulation of hyperubiquitylated p53. Conversely, interference with p53 hyperubiquitylation in NB cells by inhibiting the p53-Hdm2 interaction efficiently relocates p53 to the nucleus and reactivates p53's transcriptional and apoptotic functions. Thus, targeting p53 hyperubiquitylation by downregulating $\mathrm{Hdm} 2$ or by reactivating HAUSP may prove useful in the therapy of NB.

\footnotetext{
${ }^{1}$ Department of Pathology, State University of New York at Stony Brook, Stony Brook, NY, USA and ${ }^{2}$ Department of Cell Biology, University Medical Center Utrecht, Utrecht, The Netherlands

${ }^{*}$ Corresponding author: UM Moll, Department of Pathology, Stony Brook University, Nichols Road, Stony Brook, NY 11794-8691, USA. Tel: + 631 444 2459;

Fax: + 631444 3424;

E-mail: umoll@notes.cc.sunysb.edu

Keywords: neuroblastoma; p53; ubiquitylation; sequestration; cytoplasm; Nutlin

Abbreviations: Camp, camptothecin; $\mathrm{Cr}$, crude; Cyt/C, cytoplasmic (fraction); HAUSP, herpes virus-associated ubiquitin-specific protease; IP, immunoprecipitation; $\mathrm{K}$, lysine residue; Mito, mitochondrial (fraction); Mut, mutant; NB, neuroblastoma; Nuc/N, nuclear (fraction); PARC, parkin-like ubiquitin ligase; WB, western blot; Wt, wildtype; Ub, ubiquitin

Received 23.10.06; revised 19.2.07; accepted 20.2.07; Edited by V De Laurenzi; published online 23.3.07
} 


\section{Results}

Wild-type p53 is hyperubiquitylated in NB. As p53 ubiquitylation is a prerequisite for nuclear export, we analyzed the ubiquitin status of endogenous p53 in a set of human NB cell lines. IMR32, SKN-SH, CHP134 and LAN5 were previously characterized to harbor wild-type p53 that is significantly sequestered in the cytoplasm. ${ }^{5,8,11,26} \mathrm{NB}$ lines were compared to non-NB human cancer lines with either nuclear wild-type p53 (HCT116, U2OS, RKO) or nuclear mutant p53 (MDA 468, T47D, MDA 231), using immunoblot analysis normalized for equal amounts of non-ubiquitylated p53. As ubiquitylated p53 is normally rapidly degraded via the proteasome pathway and therefore difficult to detect, cells were treated with proteasome inhibitor ALLN. As shown in Figure 1a, NB-derived wtp53 is markedly more ubiquitylated in all four NB lines, as indicated by the characteristic 'ladder' recognized by p53-specific antibody D01. In contrast, non-NB wild-type cells have much lower levels of ubiquitylated p53, whereas mutant p53 is almost completely non-ubiquitylated. Of note, this phenotype of p53 hyperubiquitylation in NB cells is readily detectable even without proteasome inhibition (Figure 1b). To confirm further that the characteristic 'ladder' of higher molecular weight bands detected by D01 represent ubiquitylated p53 species, we immunoprecipitated for p53 and blotted comparable amounts of non-Ub p53 with the ubiquitin-specific antibody P4D1 or D01. As expected, P4D1, although a rather poor antibody (as all available anti-Ub antibodies), generates a 'ladder' pattern similar to DO1 in NB but not in non-NB cells (Figure 1c).

Importantly, proteasome inhibition significantly increases the level of ubiquitylated p53 in both non-NB and NB cells. In NB cells, ALLN protection generates an intense continuous smear of p53 species that consist of high molecular weight polyUb-p53 (>100 kDa) and smaller (multi)monoUb p53 species (Figure 1d). This indicates that the proteasomal degradation pathway per se is functional in NB. In support, levels of two other proteins - Hdm2 (discussed later in Figure $3 \mathrm{a}$ ) and $\mathrm{I} \kappa \mathrm{B} \alpha$ (as published previously; data not shown), ${ }^{10}$ which are also degraded via the ubiquitin-proteasome pathway, are within normal range in NB cells. Thus, accumulation of hyperubiquitylated p53 in NB cells is not due to a general proteasomal dysfunction. To demonstrate further that the predominant Ub-p53 species consitutively present in NB cells under normal conditions is actually (multi)mono ubiquitylated, we used the non-branchable ubiquitin mutant UbKO. $^{34}$ In the presence of UbKO, p53-null H1299 cells transfected with p53 show the typical (multi)monoUb p53 laddering pattern, irrespective of the presence of ALLN (Figure 1d right, lanes 1-4). A similar endogenous p53 pattern is found in NB cells in the absence of ALLN (lanes 5 and 6), indicating that (multi)mono ubiquitylation indeed represents the predominant form of hyperubiquitylation observed in NB.

Of note, the pool of hyperubiquitylated p53 is rather resistant to DNA damage (Figure 1e). Normally, genotoxic stress induces Hdm2 degradation and a series of phosphorylations on both p53 and Hdm2, disrupting the p53-Hdm2 complex. This rapidly stabilizes p53 and renders it active. ${ }^{27,28}$
However, when comparing similar amounts of stress-stabilized p53 (Figure 1e, 'short' exposure), p53 from NB cells, whereas its ubiquitination does decrease somewhat, remains significantly hyperubiquitylated compared to non-NB cells.

Hyperubiquitylated p53 in NB is cytoplasmic and does not shuttle. Since p53 ubiquitylation by $\mathrm{Hdm} 2$ induces p53 nuclear export ${ }^{18-20}$ we analyzed the hyperubiquitylation phenotype of p53 with respect to subcellular localization. As expected, LAN5 NB cells display diffuse cellular p53 staining, indicating significant cytoplasmic sequestration (Figure 2a left). Importantly, subcellular fractionation shows that the cytoplasm of NB cells preferentially contains hyperubiquitylated p53, whereas the nucleus mainly contains non-ubiquitylated p53 (Figure 2a right and data not shown). In contrast, wild-type p53 expressing colon carcinoma cells (RKO), with lower levels of p53 ubiquitylation than NB cells (Figure 1), exhibit mainly (but not exclusively) nuclear p53 localization (Figure 2a left) and much lower levels of hyperubiquitylated p53 in the cytoplasm (Figure 2a right). Finally, MDA 468 breast cancer cells that contain almost exclusively non-ubiquitylated p53 show strictly nuclear p53 staining of very high intensity. Thus, cytoplasmic sequestration of p53 in NB strictly correlates with p53 hyperubiquitylation.

In addition to p53's transcriptional role, stress-induced p53 translocation to mitochondria which induces outer mitochondrial membrane permeabilization is a common early component in p53-mediated apoptosis in normal and transformed cells. $^{29}$ Importantly, in contrast to non-NB cells that contain functional wtp53 such as RKO and ML1, both NB lines tested (CHP134 and LAN5) are markedly impaired in their ability to undergo stress-induced mitochondrial translocation (Figure 2b). This further supports the notion that cytoplasmically accumulated p53 in NB is inactive in mediating apoptosis.

We next asked whether ubiquitylated p53 is still able to shuttle. However, treatment of NB cells with the CRM 1 nuclear export inhibitor leptomycin B results in nuclear accumulation of only non-ubiquitylated p53, whereas all ubiquitylated p53 species remain sequestered in the cytoplasm (Figure 2c, Supplementary Figure 1). The lack of ubiquitylated p53 species in the nucleus is neither due to enhanced proteasomal degradation, nor due to enhanced HAUSP-mediated deubiquitylation in the nucleus, because the differential cytoplasmic/nuclear p53 ubiquitylation pattern upon LMB is similar in the absence or presence of proteasomal inhibition (Figure 2c), and the levels of HAUSP are not elevated in the nuclear fraction (Figure $2 c$ ). Instead, as only non-ubiquitylated but not ubiqutinated p53 accumulates in the nucleus upon export blockade, it might reflect the possibility that p53 ubiquitylation inhibits nuclear re-import. This notion is supported by the finding that all NB cells constitutively have a much higher fraction of p53 complexed with $\beta$-importin, a component of the nuclear import pore, than do non-NB cells (Figure 2d). Taken together, we speculate that hyperubiquitylated p53 cannot be re-imported because it is either arrested in a non-productive complex at the import pore or is inhibited from binding to the functional pore by soluble $\beta$-Importin. 

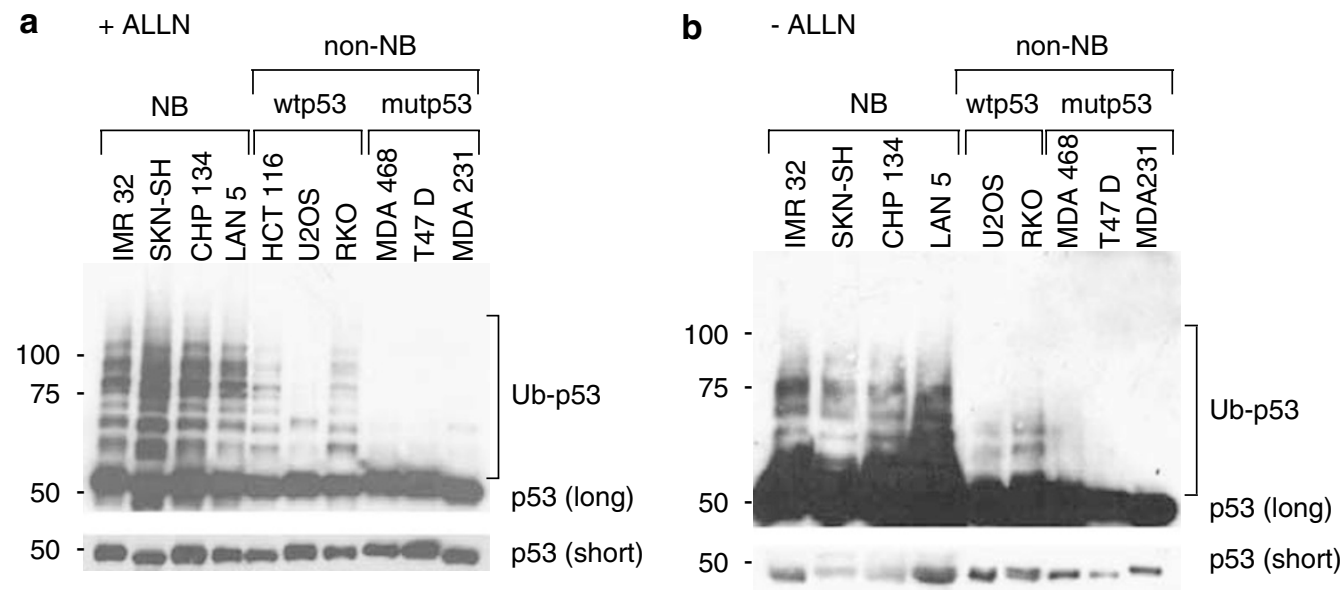

C

IP: CM1
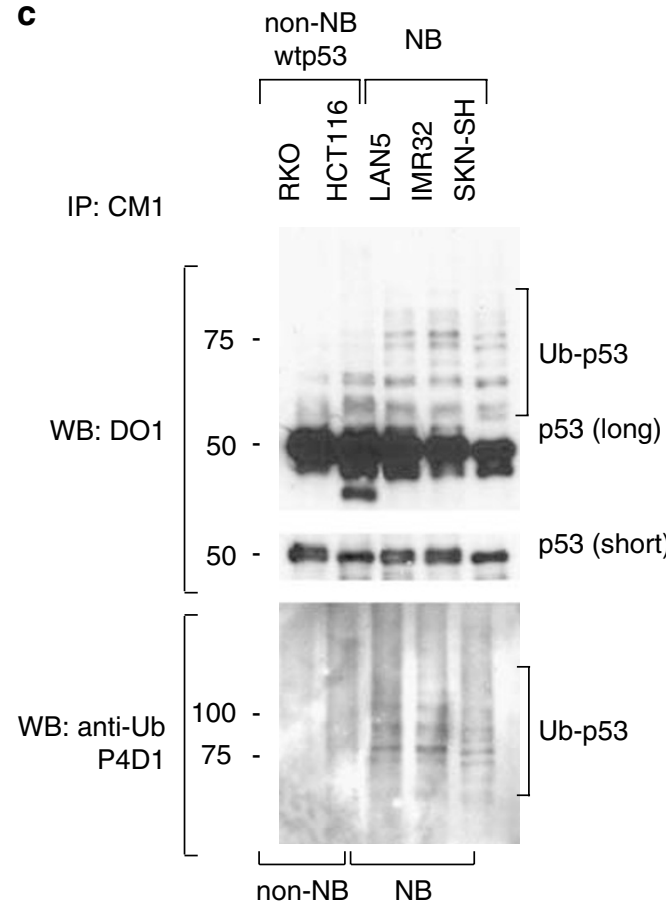

d

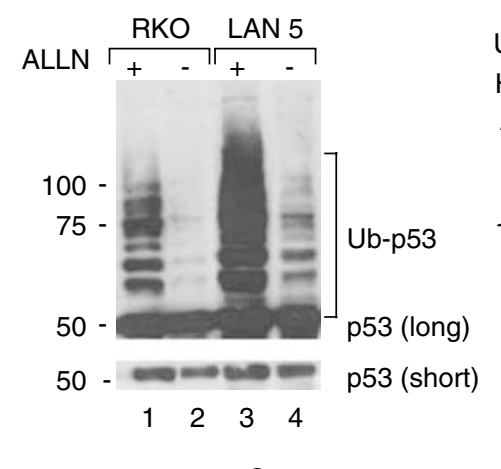

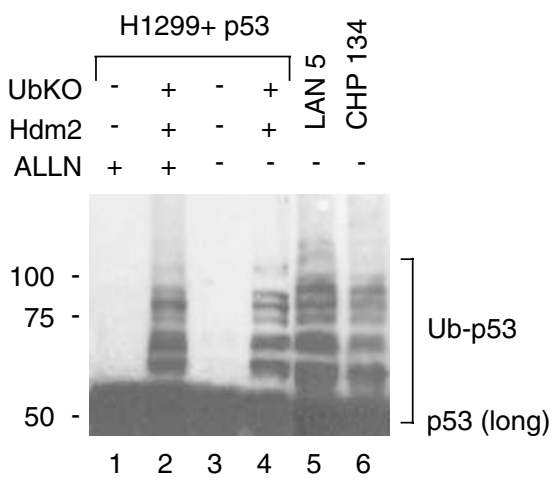

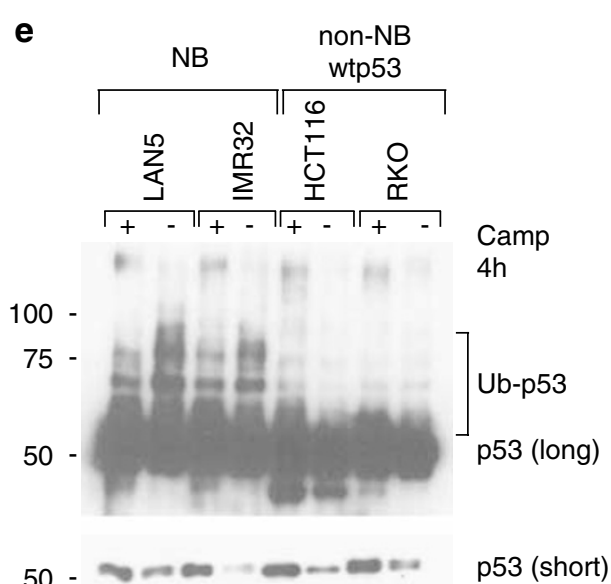

50

p53 (short)

Figure 1 Wild-type p53 is hyperubiquitylated in NB. (a) NB lines were compared to non-NB human cancer lines with either nuclear wild-type p53 (HCT116, U2OS, RKO) or nuclear mutant p53 (MDA 468 (p53R273H), T47D (p53L194F), MDA 231 (p53R280K)). NB and non-NB human cancer cell lines were treated with the proteasome inhibitor ALLN $3 \mathrm{~h}$ before harvesting to prevent proteasomal degradation. p53 loading was normalized for comparable amounts of non-ubiquitylated p53 per lane and blotted with DO1. Non-NB tumor cell lines with functional nuclear wtp53 exhibit low levels of p53 ubiquitylation that varies within a certain range (normal controls). Mutant p53-harboring cell lines with their highly elevated levels of mutant protein have even lower levels of p53 ubiquitylation, for reasons that are not clear (although at face value this could explain their elevated p53, they have normal amounts of Hdm2 (Figure 3a)). In contrast, all four NB lines, which harbor moderately elevated levels of wild-type p53 that is significantly sequestered in the cytoplasm and functionally impaired, constitutively express hyperubiquitylated p53. (b) p53 hyperubiquitylation in NB cells is readily detectable without proteasome inhibitor. Short and long exposures are shown. (c) Immunoprecipitation of p53 from NB and non-NB cells followed by blotting with either p53-specific (D01) or ubiquitin-specific (P4D1) antibody. A similar characteristic ladder as detected by D01 is recognized by P4D1 (albeit not band-for-band) only in NB cells. (d) Left. The proteasome inhibitor ALLN stabilizes the ubiquitylated p53 species in non-NB (RKO) and in NB (LAN5) cells, although there is a stark difference in the amount of ubiquitylation at baseline and after ALLN. Immunoblot normalized for p53 loading. Right. NB cells constitutively exhibit stable multimono-ubiquitylates p53. p53 null H1299 cells were transiently transfected with wtp53 with or without UbKO and Mdm2. Three hours after transfection, cells were treated with ALLN where indicated. Immunoblot normalized for p53 loading. The ladder patterns in lanes 5 and 6 correspond to multimono-ubiquitylated p53 in lane 4. (e) p53 remains hyperubiquitylated in NB cells upon stress. NB and nonNB cells were treated with $5 \mu \mathrm{M}$ camptothecin or mock for $4 \mathrm{~h}$. One milligram of total protein each was immunoprecipitated with a 1:1 cocktail of p53-specific D01/PAb1801 antibodies conjugated agarose beads and blotted with p53-specific CM1. (a-e) Immunoblots with p53-specific D01 monoclonal antibody 
p53 hyperubiquitylation in NB is neither caused by Hdm2 overexpression nor by HAUSP downregulation, but owing to impaired p53-HAUSP interaction. Next, we addressed the underlying cause of p53 hyperubiquitylation in NB. One reason could be increased ubiquitylation by $\mathrm{Hdm} 2$, especially as the Hdm2 gene was reported to be amplified in some NB cell lines. ${ }^{30}$ However, in all NB lines used here Hdm2 levels are not elevated, but either lower (LAN5 and SKN-SH) or comparable (CHP134 and IMR32) to non-NB control cells (Figure $3 \mathrm{a}$ ). Thus, Hdm2 overexpression does not appear to be the cause of p53 hyperubiquitylation. More importantly, we found comparable amounts of p53-Hmd2 complexes in NB cells and non-NB controls (Figure $3 b$ ). Another reason for p53 hyperubiquitylation might be decreased deubiquitylation by HAUSP. Yet, total protein levels of HAUSP are normal in NB cells compared to non-NB controls (Figure 3a). However, functional interaction between HAUSP and p53 is impaired, as shown by co-immunoprecipitations for p53 and HAUSP. All NB lines exhibit significantly less p53-HAUSP complexes than control lines, resulting in p53 hyperubiquitylation (Figure 3c). On the other hand, the subcellular distribution of HAUSP in NB and non-NB cells is identical, excluding aberrant HAUSP distribution as the reason for HAUSP impairment (Figure $3 \mathrm{c}$ bottom). In contrast, although $\mathrm{Hdm} 2$ is also a major substrate of HAUSP, the HAUSP-Hdm2 complex formation in NB cells is within normal range, compared to non-NB controls (Figure 3d). As shown previously, ${ }^{8}$ the cytoplasmic protein PARC is overexpressed in NB cells (Figure $3 e$ left) and interestingly, the amount of PARC-p53 complexes is higher in NB than in non-NB cells (Figure 3e right). Moreover, the fact that under stress conditions when p53-Hdm2 interactions no longer play a role, NB-derived p53 largely remains hyperubiquitylated also argues for a defect in deubiquitylation rather than a defect in Hdm2-mediated ubiquitylation (Figure 1e). Together, a defect in HAUSP. mediated deubiquitylation appears to be responsible for p53 hyperubiquitylation and sequestration in NB.

HAUSP downregulation in non-NB cells causes a
phenotype that also results in cytoplasmic accumulation of hyperubiquitylated p53. To confirm this hypothesis, we reasoned that a switchable impairment of the p53-HAUSP interaction in non-NB cells should induce a NB phenotype. To this end, we used the human colon carcinoma cell line LS126 that stably expresses Dox-inducible HAUSP shRNA. ${ }^{25}$ In this tightly regulated system of the dynamic HAUSP-Hdm2-p53 triangle, HAUSP downregulation upon doxycyclin is nearly complete (Figure $4 a$ bottom). As Hdm2 is a major substrate of HAUSP, this results in a marked drop (but not complete abolition) in Hdm2 levels owing to increased Hdm2 autoubiquitylation and self-destruction (Figure 4a), which in turn causes stabilization of nonubiquitylated p53 (Figure $4 \mathrm{a}$ bottom), in agreement with previous reports. ${ }^{23,24}$ On the other hand, the remaining Hdm2 still ubiquitylates p53 and these Ub-p53 species, despite ongoing degradation, now net accumulate due to HAUSP deficiency (Figure $4 a$ top). Hence, Dox-treated LS126 cells resemble the p53 and p53/HAUSP status of NB cells. Of note, p53 ubiquitylation induced by HAUSP ablation is accompanied by p53 accumulation in the cytoplasm (Figure 4b). Cell fractionation shows a net increase of p53 in the cytoplasmic fraction upon HAUSP ablation, entirely accounting for the increase of total cellular p53 levels upon Dox treatment (Figure 4b top). In contrast, nuclear p53 levels do not increase but slightly drop upon Dox. Immunofluorescence confirms this shift to the cytoplasm. Untreated LS126 cells show predominantly nuclear p53 staining, whereas Dox-treated cells exhibit a cytoplasmic p53 staining, in addition to nuclear staining (Figure 4b).

Interference with p53 hyperubiquitylation in NB results in relocalization from the cytoplasm to the nucleus and reactivation of p53 transcriptional and apoptotic function. Conversely, we reasoned that the NB phenotype should be reversible to a non-NB phenotype by decreasing p53 hyperubiquitylation in NB cells. To this end, we used Nutlin $3 a$, a highly specific and effective small molecule $\mathrm{Hdm} 2$ inhibitor $^{31}$ to disrupt directly the p53-Hdm2 interaction in NB cells. Indeed, when CHP134 NB cells were treated with Nutlin $3 a$, the level of p53 ubiquitylation decreased in a dosedependent fashion with increasing Nutlin concentrations (Figure 5a), whereas total cellular levels of p53 increased (Figure 5b). Of note, following the degree of p53 deubiquitylation, CHP134 cells show a steadily increasing shift in p53 distribution from the cytoplasm to the nucleus with increasing Nutlin concentrations (Figure 5a). As expected, translocation of $p 53$ to the nucleus is followed by reactivation of p53 transcriptional function, as assessed by target genes Hdm2 and p21, and p53-mediated apoptosis, as indicated by caspase-3 activation and PARP cleavage in a dosedependent manner (Figure 5b). Moreover, Nutlin synergizes with camptothecin's effect in inducing NB cell apoptosis, as indicated by PARP cleavage and TUNEL assay, whereas camptothecin alone has only minimal effect (Figure 5b middle and right and data not shown).

Finally, to further support the Nutlin results, we used a previously described, derivative LAN5 NB line, that stably expresses a C-terminal fragment of p53 (aa 305-393), which lacks the $\mathrm{N}$-terminal Hdm2 binding site and functions as a dominant-negative peptide by engaging in mixed tetramers with endogenous p53. ${ }^{9,11}$ Of note here, p53 in the derivative cells is highly stabilized and virtually non-ubiquitylated. In contrast, parental LAN5 cells have lower levels of p53, of which a significant portion is ubiquitylated (Figure $5 \mathrm{c}$ left). The decrease in p53 ubiquitylation in LAN5(305-393) cells is due to a lack of p53-Hdm2 interaction, as shown by immunoprecipitation (Figure 5c middle). Interestingly, LAN5 parental cells exhibit a detectable interaction between p53 and the nuclear export receptor CRM 1 indicating export, whereas such a complex is undetectable in LAN5(305-393) cells (Figure 5c right). Finally, consistent with the latter data and the Nutlin results, lack of p53 ubiquitylation in LAN5(305-393) cells correlates with nuclear accumulation of $\mathrm{p53}$, whereas parental cells with hyperubiquitylated p53 display significant cytoplasmic distribution (Figure 5c). Thus, interfering with hyperubiquitylation of p53 in NB cells, whether by Nutlin 3a or by mixed tetramers, yields the same results, that is, relocalization of p53 from the cytoplasm to the nucleus. Moreover, in the case of Nutlin, this induces reactivation of p53 function. 


\section{Discussion}

Our data show that aberrant hyperubiquitylation of wild-type p53, owing to an impaired p53-HAUSP interaction, contributes to cytoplasmic sequestration and attenuation of p53 function in NB cells. Conversely, interference with p53 hyperubiquitylation by inhibiting the p53-Hdm2 interaction efficiently relocates p53 to the nucleus and reactivates p53's transcriptional and apoptotic function.
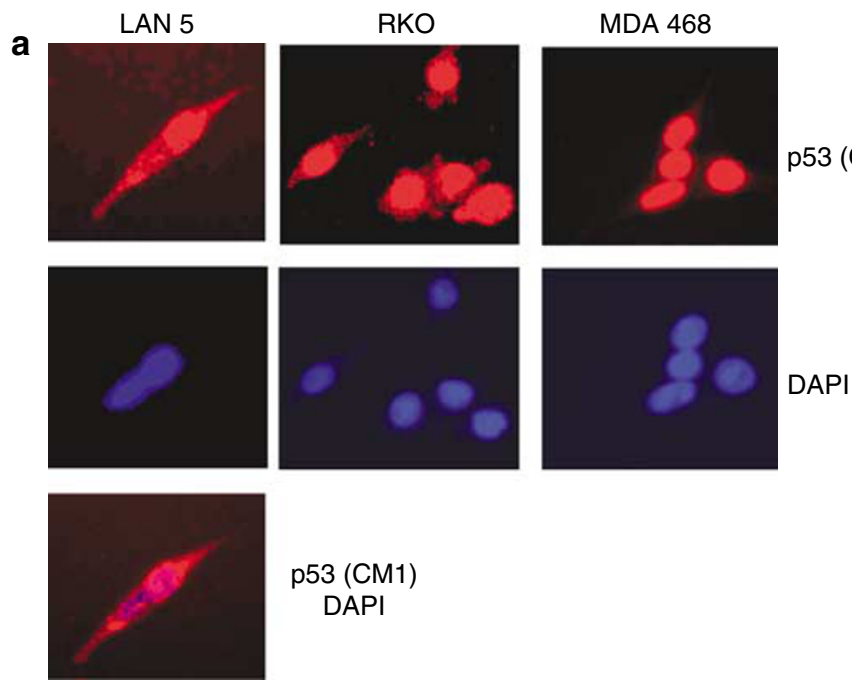

p53 (CM1)

DAPI
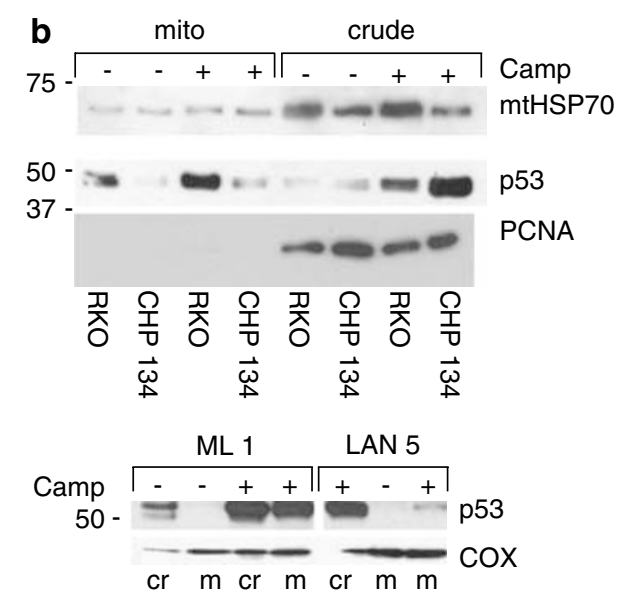

c $\mathrm{SKN}-\mathrm{SH}$

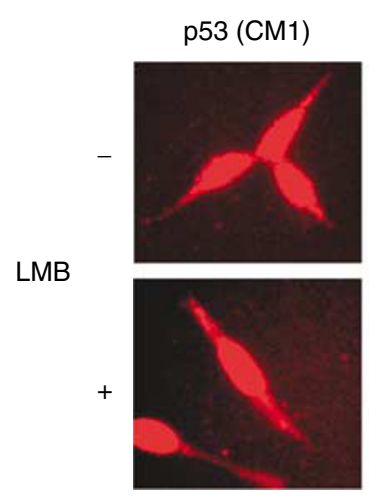

p53 (CM1)

DAPI
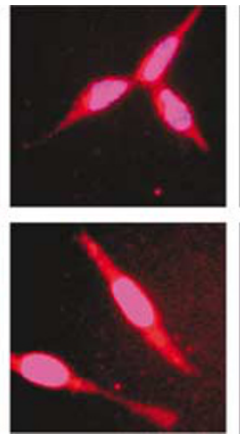

DAPI
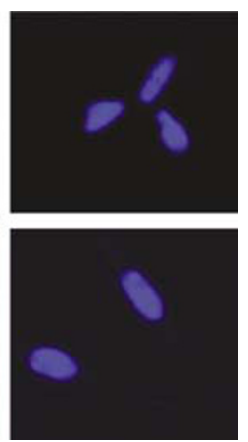

d

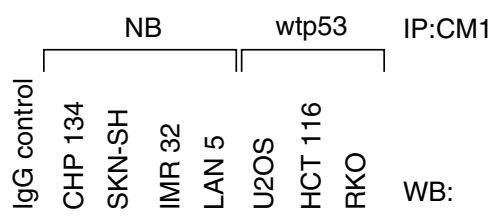

$50-$

$100-$

$75-$

p53

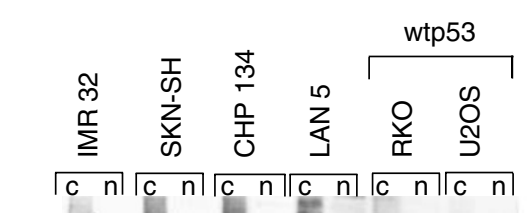

$100-$

$75-$

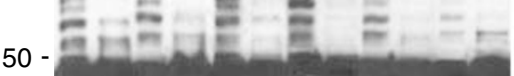

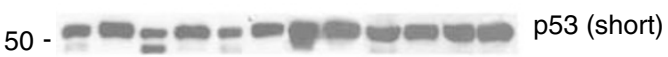

$100-\ldots-\ldots$ HSP 90

$75-----\operatorname{HDAC} 1$

50 -

$\beta$-importin

SKN-SH

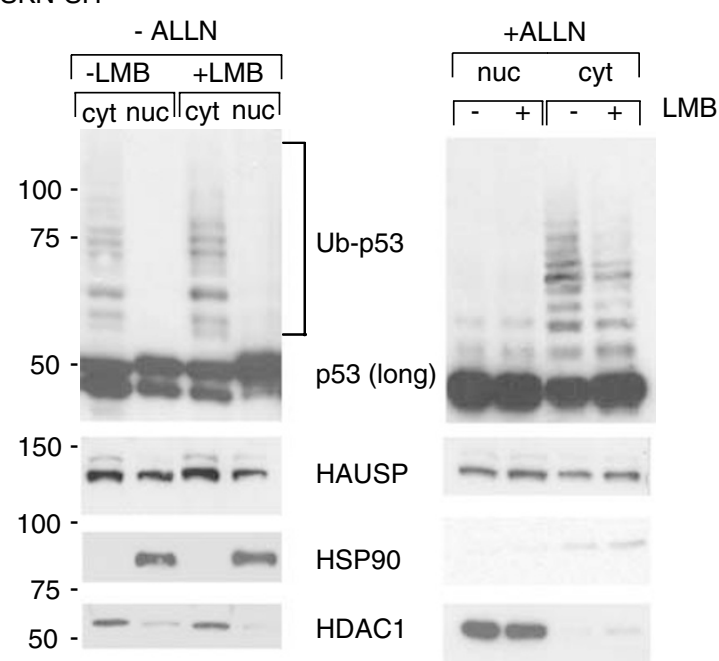


Our findings can be integrated with previous reports in the following way: in the NB cells studied here, the dynamics regulating p53 ubiquitylation is disturbed, which upsets the balance and initiates a chain of events. A core defect is the impaired interaction between p53 and HAUSP, leading to defective deubiquitylation of p53. At the same time (and differing from HAUSP ablation experiments), ${ }^{23,24}$ the Hdm2HAUSP interaction and overall $\mathrm{Hdm} 2$ protein levels are normal. The net effect is p53 hyperubiquitylation, consisting of a spectrum of polyUb-p53 and (multi)monoUb-p53 species. PolyUb-p53 is unstable and rapidly degraded by proteasomes. On immunoblots it is therefore not routinely detectable unless in very favorable circumstances using proteasome inhibitors (see Figure 1c). Degradation of p53 takes place in the cytoplasm as well as in the nucleus. ${ }^{32}$ In contrast, (multi)monoubiquitylated proteins in general are stable, as efficient proteasomal degradation minimally requires a multiubiquityl chain at least four subunits long per single lysine residue. ${ }^{33}$ Thus, (multi)monoUb-p53 species in NB are stable and accumulate. Furthermore, they undergo preferential nuclear export and, once exported, appear unable to re-enter the nucleus. This interpretation is consistent with findings by $\mathrm{Li}$ et al. ${ }^{21}$ in non-NB cells that monoubiquitylated p53 is stable and subject to nuclear export, whereas polyubiquitylated p53 is rapidly degraded in the nucleus. Hence, the decrease of p53 deubiquitylation owing to impaired HAUSP interaction in NB cells results in a net increase of (multi)monoubiquitylated p53 species, which are relatively resistant to proteasomal degradation and accumulate in the cytoplasm. The end result is that a substantial portion of the available p53 is diverted from the activatable pool (Figure 6).

Mechanistically it is still unclear, how p53 ubiquitylation affects its subcellular localization. It was suggested that p53 ubiquitylation promotes nuclear export because it changes the conformation of its $\mathrm{C}$ terminus, thus revealing the nuclear export signals to the export machinery. ${ }^{34}$ Indeed, we find enhanced interaction between p53 and CRM 1 in parental LAN5 cells which contain ubiquitylated p53 in the cytoplasm, as compared to non-ubiquitylated p53 in LAN5(305-393) cells which contain exclusively nuclear p53 (Figure 5c). However, only non-ubiquitylated p53 can accumulate in the nucleus upon LMB treatment, whereas ubiquitylated p53 species remain sequestered in the cytoplasm (Figure $2 \mathrm{~b}$ ). This observation prompted us to hypo- thesize that in addition to hyperactive nuclear export, hyperubiquitylation might also impair nuclear re-import by neutralizing the positive charge of lysines which are critical for recognition by the nuclear import machinery. Intriguingly, all six- known lysine residues that have been identified as targets for ubiquitylation locate within the nuclear localization signals NLS II and NLS III.

Mutational analyses established the importance of the p53 $\mathrm{C}$ terminus in Hdm2-mediated ubiquitylation. ${ }^{16,17}$ In line with this, we previously reported a covalent modification of the p53 $\mathrm{C}$ terminus in NB cell lines, as indicated by NB-specific masking of the modification-sensitive epitope of monoclonal p53 antibody PAb421 (residues 372-382) and a dramatic shift in immune-isoelectrofocussing in the p53 isoform profile to a ladder of hyperacidic forms (Supplementary Figure 2). ${ }^{10}$ Given the current data, these isoforms can now be identified as the hyperubiquitylated p53 species described here. By forming isopeptide bonds with the $\varepsilon$-amino groups, ubiquitylation neutralizes the positively charged lysine residues on the $C$ terminus, increasing protein acidity.

The precise mechanism underlying the impaired p53HAUSP interaction in NB cells remains unclear but multiple lines of evidence suggest that the $p 53 \mathrm{C}$ terminus plays a role. The binding site for HAUSP on p53 maps within aa 359$367 .{ }^{35}$ Interestingly, like HAUSP, PARC also binds to the C terminus of p53 (mapped to aa 290-393) ${ }^{8}$ and therefore might compete out HAUSP. Moreover, we show that NB cells overexpress PARC and exhibit a higher amount of PARC-p53 complexes than non-NB cells (Figure $3 e$ ). In line with our results, Nikolaev et al. ${ }^{8}$ reported that PARC is highly expressed in NB lines compared to normal human brain tissue or human glioma cell lines and sequesters p53 in the cytoplasm of NB lines, including the LAN5 and SKN-SH lines used here. Furthermore, when PARC levels were knocked down by siRNA in SKN-SH cells, p53 underwent nuclear relocalization, induction of p21 and restoration of apoptotic function. ${ }^{8}$ Of note, although PARC possesses intrinsic ubiquitin ligase activity, it failed to induce ubiquitylation of p53, thus it plays no role in p53 ubiquitylation. ${ }^{8}$ On the other hand, as the enhanced p53-PARC complex formation in NB involves the p53 $C$ terminus, this strongly supports a mechanistic role for PARC in the impairment of the p53HAUSP interaction in NB cells. However, we cannot completely exclude other mechanisms. For example, the p53 C

Figure 2 Hyperubiquitylated p53 in NB is cytoplasmic and does not shuttle. (a) Left. p53 staining of NB (LAN5) and two non-NB cell lines with p53-specific CM1 antibody (red). The state of p53 ubiquitylation closely correlates with p53 nuclear/cytoplasmic localization. LAN5 cells (a representative cell is shown) with p53 hyperubiquitylation display diffuse cellular p53 staining with absence of exclusive nuclear staining and significant cytoplasmic p53 staining of moderate intensity in all cells. In contrast, RKO cells (increased levels of functional wtp53) with normal ubiquitylation exhibit mainly nuclear p53 localization, and MDA 468 cells (highly stabilized mutant p53) with barely detectable ubiquitylation exhibit exclusively nuclear localization. DAPI staining to indicate nuclei. Right. nuclear-cytoplasmic fractionation of NB and non-NB cells. Immunoblot as indicated. Cytoplasmic p53 is highly ubiquitylated in contrast to nuclear p53. HSP 90 as cytoplasmic marker and HDAC1 as nuclear marker. (b) NB cells (CHP134 top and LAN5 bottom) are markedly impaired in their ability to undergo stress-induced mitochondrial translocation. RKO and ML1 cells are positive controls. Equal amounts of crude lysates (crude or ' $c r$ ') and purified mitochondria (mito or ' $\mathrm{m}$ ') ( $4 \mu \mathrm{g}$ per lane) from cells untreated or treated with Camptothecin ( $5 \mu \mathrm{M}$ for $4 \mathrm{~h}$ ) were immunoblotted. Mt hsp70 was used as loading control for RKO and CHP134, and COX IV was used for ML1 and LAN5. Purity from nuclear contamination was verified by re-blotting with PCNA antibody. (c) Treatment of SKN-SH NB cells with the nuclear export inhibitor leptomycin B (LMB) results in accumulation of non-ubiquitylated p53 in the nucleus indicating shuttling, whereas ubiquitylated p53 remains sequestered in the cytoplasm. Left: Immunofluorescence with CM1 antibody. Note that although LMB induces nuclear accumulation, the cytoplasm retains its faint positive staining. DAPI counterstaining. Right. Nuclear-cytoplasmic fractionation of SKN-SH cells $+/-$ LMB for $6 \mathrm{~h}$ with (right) and without ALLN (left). Immunoblot with DO1 antibody, HSP 90 as cytoplasmic marker and HDAC1 as nuclear marker. The same results were obtained with LAN5 cells (Supplementary Figure 1). (d) NB cells with hyperubiquitylated p53 constitutively have a higher fraction of p53 in complex with the nuclear import pore than do non-NB cell types. Immunoprecipitations of comparable amounts of p53 from cell lysates with CM1 were probed with $\beta$-importin 


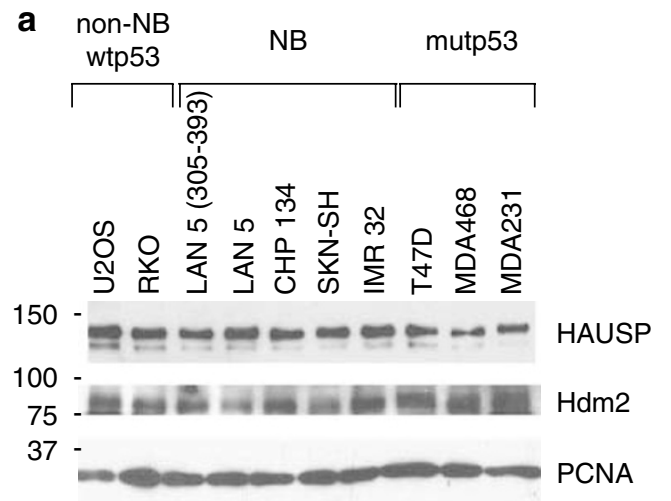

$\begin{array}{lcc}\text { b } & \text { IP:Hdm2 } & \text { non-NB } \\ \text { WB: p53 } & \text { wtp53 } & \text { NB }\end{array}$

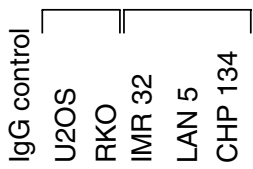

$50-$ p53

$100-$

75 -

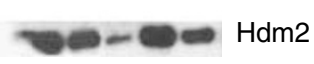

d

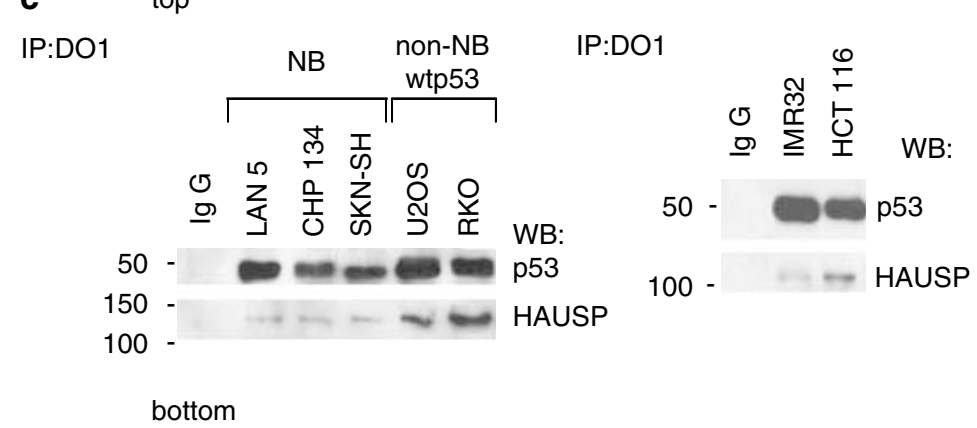

$$
\begin{aligned}
& \text { IP: HAUSP IP } \begin{array}{c}
\text { crude } \\
\text { extract }
\end{array}
\end{aligned}
$$

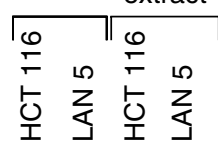

150 -

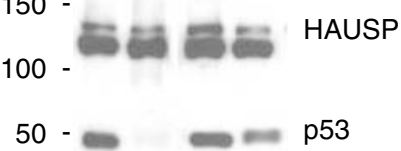

WB:
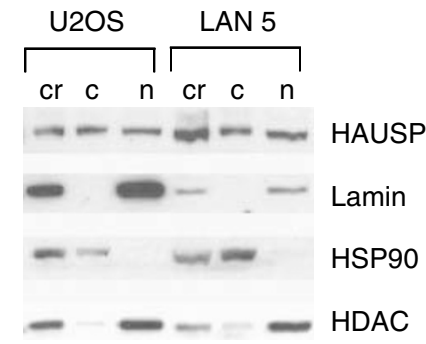

150 -

$100-$

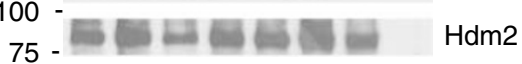

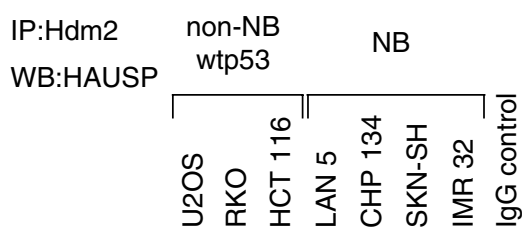

HAUSP

e

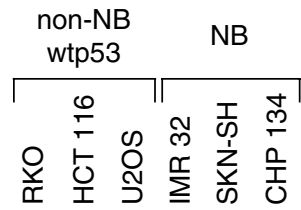

WB:
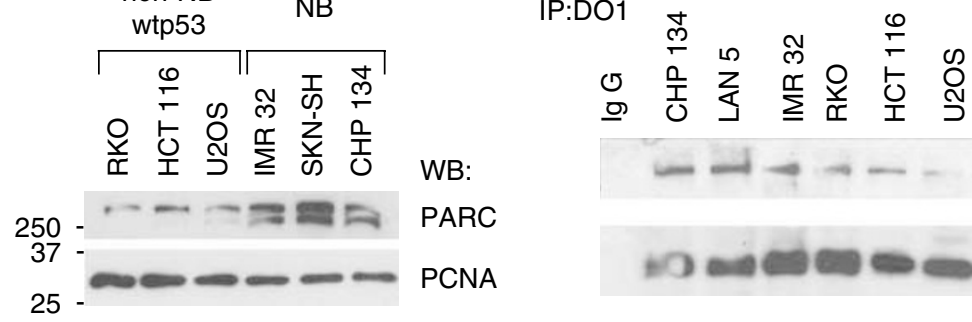

WB:

PARC

Figure 3 NB cells have normal Hdm2 and HAUSP protein levels, but the p53-HAUSP interaction is impaired. (a) NB and non-NB cell types have comparable levels of $\mathrm{Hdm} 2$ and HAUSP. Some variation from cell line to cell line is noted. Immunoblots for HAUSP and Hdm2 using $10 \mu \mathrm{g}$ of protein per lane. PCNA as loading control. (b) NB cells have normal interactions between p53 and Hdm2. Immunoprecipitation from NB and non-NB cells for Hdm2 and blotted for p53 and Hdm2. (c) The p53-HAUSP interaction is impaired in NB. Top: immunoprecipitation for p53 (D01) with $1 \mathrm{mg}$ of total cell lysate each was immunoblotted for HAUSP. NB cell lines exhibit less p53-HAUSP complexes than non-NB wtp53 cancer cell lines. IgG as negative control. Bottom left. Immunoprecipitations for HAUSP from wtp53-expressing colon cancer (HCT116) and NB cells (LAN5) with $2 \mathrm{mg}$ of total cell lysate each were immunoblotted for p53 with D01 (two left lanes). The two right lanes are direct immunoblots of $10 \mu \mathrm{g}$ of crude cell lysates to show comparable p53 and HAUSP content for these lines. Bottom right. The subcellular distribution of HAUSP in NB is identical to that in non-NB cells. Equal amounts of crude lysates ('cr'), cytoplasm ('c') and nuclear ('n') (5 $\mu \mathrm{g}$ per lane) from U2OS and LAN5 cells were immunoblotted with HAUSP antibody. HSP 90 as cytoplasmic marker, and HDAC and lamin as nuclear marker. (d) NB cells have normal interactions between Hdm2 and HAUSP. Immunoprecipitation from NB and non-NB cells for Hdm2. Immunoblot for HAUSP and Hdm2 to ensure equal Hdm2 loading. (e) Left. NB cell lines have elevated levels of the cytoplasmic anchor protein PARC that runs as two species. Immunoblot of total cell lysates from NB and non-NB cells with polyclonal anti-PARC. PCNA is loading control. Right. NB cells contain more p53-PARC complexes than non-NB cells. Immunoprecipitation for p53 (D01) with $2 \mathrm{mg}$ of total cell lysate each was immunoblotted for PARC. WB input was normalized for similar amounts of p53 


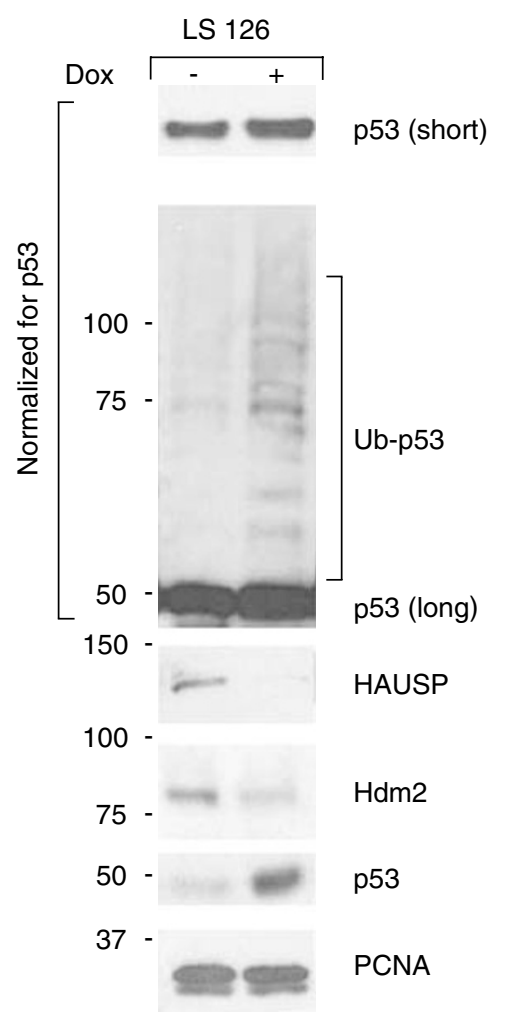

LS 126
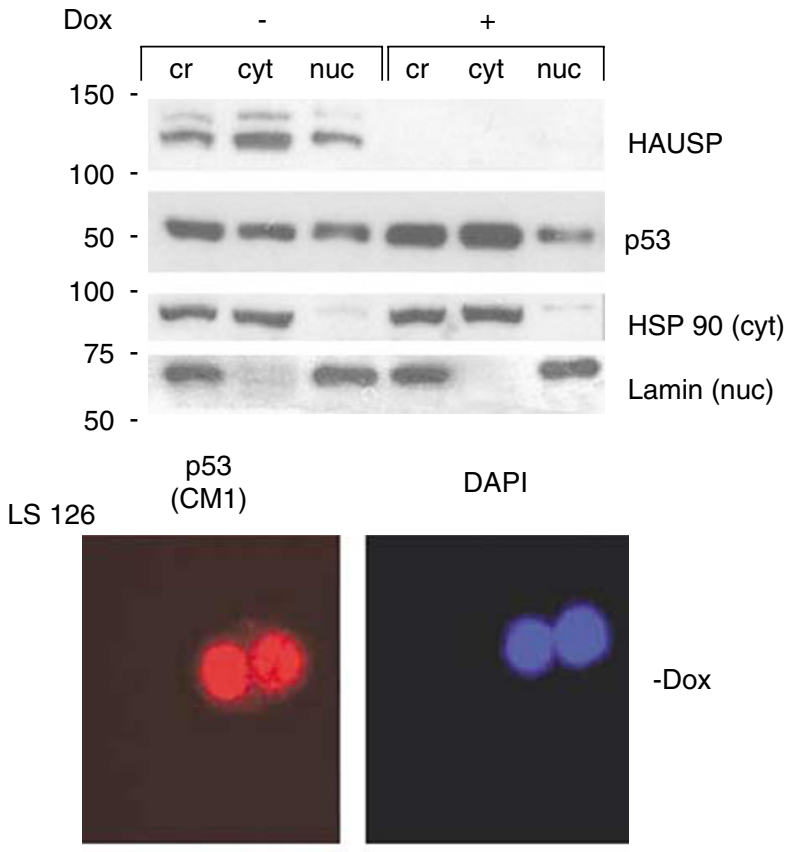

DAPI
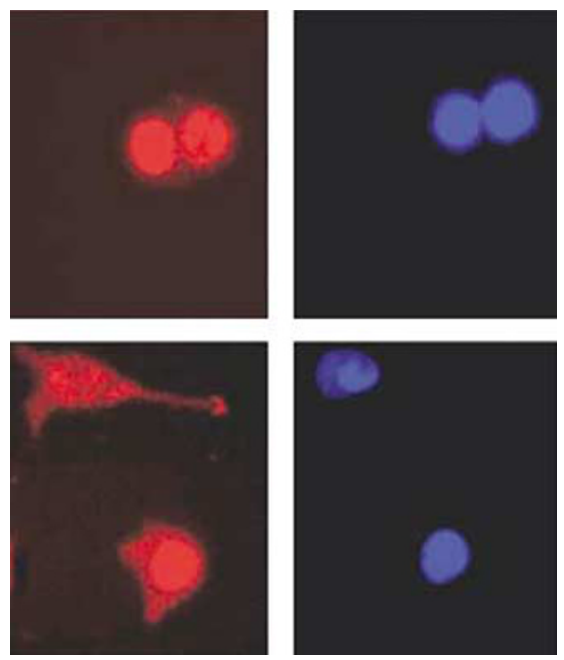

-Dox

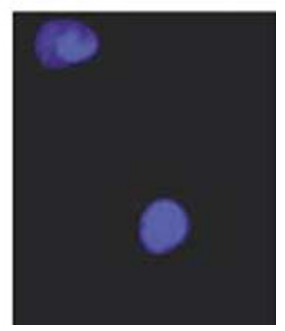

+ Dox

Figure 4 HAUSP downregulation in non-NB cells causes a phenotype that also results in cytoplasmic accumulation of hyperubiquitylated p53. (a) Immunoblot analysis of LS126 cells stably expressing Dox-inducible HAUSP shRNA. Upon doxycyclin, HAUSP levels are significantly downregulated, resulting in Hdm2 destabilization and p53 stabilization (lower four panels, equal protein loading as indicated by PCNA). Normalization for equal amounts of non-ubiquitylated p53 loading shows that p53 is more ubiquitylated in Dox treated than in untreated cells despite decreased Hdm2 levels (upper two panels). (b) Top: HAUSP downregulation increases p53 levels in the cytoplasm, entirely accounting for the increase of total cellular p53 levels upon Dox treatment (compare crude and cytoplasmic lanes). Subcellular fractionation of Dox-treated and untreated LS 126 cells and subsequent WB with $10 \mu \mathrm{g}$ of total protein as input. HSP 90 used as cytoplasmic marker and Lamin as nuclear marker. Bottom: Immunofluorescence of Dox-treated and untreated LS 126 cells with CM1 antibody for p53 staining. DAPI counterstaining

terminus can be modified at multiple sites by post-translational modifications and some of these, for example, phosphorylation of Ser 362, may affect the interaction between p53 and HAUSP. ${ }^{35}$ Moreover, HAUSP might be aberrantly modified in NB.

In another study from our lab in non-NB cells, we identify that monoubiquitylation of p53 actually promotes mitochondrial translocation. ${ }^{36}$ Interestingly, although $N B$ cells have constitutively elevated p53Ub levels in the cytoplasm, this p53Ub is completely incapable for mitochondrial p53 translocation. This clearly reveals another level of regulation for p53 mitochondrial translocation in cells. We strongly suspect that this additional level is associated with HAUSP and PARC that went array in NB cells.

The HAUSP/Hdm2 axis is required for proper p53 regulation. There is growing evidence that HAUSP downregulation might play a role in tumorigenesis, as shown in a study on non-small cell lung cancer patients. ${ }^{37}$ Our study provides further evidence that NBs suffer from a derailed Hdm2/ HAUSP regulation of $\mathrm{p53}$, either via HAUSP dysfunction (this study) or hyperactive $\mathrm{Hdm}^{3,14,30}$ both resulting in cytoplasmic sequestration due to enhanced nuclear export and possibly impaired re-import. Perhaps most importantly, this study also shows that nongenotoxic targeted drugs of the Nutlin type that are currently under development are able to reverse this dysregulated p53-Hdm2-HAUSP axis in NB. While this work was ongoing, several new studies confirm the synergism of Nutlin in chemotherapeutic control of NB cells in culture. $^{38,39}$

In sum, our findings identify p53 hyperubiquitylation as a mechanism that contributes to cytoplasmic sequestration and attenuation of $p 53$ function in NB. This $p 53$ hyperubiquitylation is due to an impaired p53-HAUSP interaction but can be reversed by inhibiting the $\mathrm{p} 53-\mathrm{Hdm} 2$ interaction. Decrease in p53 ubiquitylation resulted in nuclear retention and reactivation of p53 function in NB. Thus, we propose targeting 
aberrant $\mathrm{p} 53$ hyperubiquitylation as a promising new strategy for cancer therapy in NB management.

\section{Materials and Methods}

Cell culture and reagents. The human NB lines IMR32, SKN-SH, CHP134, LAN5 harbor moderately elevated levels of wild-type p53 that is functionally impaired and significantly sequestered in the cytoplasm., ${ }^{5,7-11}$ The human colon carcinoma cell lines RKO and HCT 116 and the osteosarcoma cell line U2OS contain mildly to moderately elevated levels of functional wtp53. The breast cancer cell lines MDA 231, MDA 468 and T47D harbour a R280K, R273K and L194F mutation in the $p 53$ gene, respectively. All cells were cultured in Dulbecco's modified Eagle's medium supplemented with 10\% fetal calf serum. LS126 cells (doxorubicin- inducible RNAi for HAUSP) were cultured as described. ${ }^{25}$ For induction of HAUSP siRNA, LS126 cells were treated with $1 \mu \mathrm{g} / \mathrm{ml}$ doxycycline for $72 \mathrm{~h}$. LAN5 (305-393) cells, which stably express the aa 305-393 C-terminal fragment of p53 containing the tetramerization domain, were described previously. ${ }^{9}$ Where indicated, cells were treated with the proteasome inhibitor ALLN $(25 \mu \mathrm{M}$, Sigma) $3 \mathrm{~h}$ before harvesting. Cells were stressed by adding Camptothecin ( $5 \mu \mathrm{M}$, Sigma) for $3 \mathrm{~h}$ before lysate preparation. Where indicated, cells were treated with leptomycin $B(10 \mathrm{nM}$ for $6 \mathrm{~h}$, BioMol Int.). Nutlin 3a (a gift from L. Vassilev, Roche) was added to the medium of CHP134 cells for $6 \mathrm{~h}$ before harvesting where indicated. Subcellular fractionation was performed using the NE-PER nuclear and cytoplasmic extraction kit (Pierce) according to the manufacturer's protocol but all extraction reagents were supplemented with a proteasome inhibitor cocktail (Roche) and ubiquitin aldehyde (Sigma) to prevent degradation and deubiquitylation.

a

CHP 134

Nutlin 3

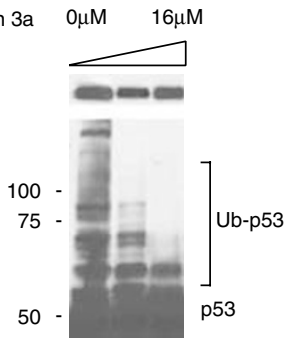

CHP 134

Nutlin 3a

$0 \mu \mathrm{M}$

$8 \mu \mathrm{M}$
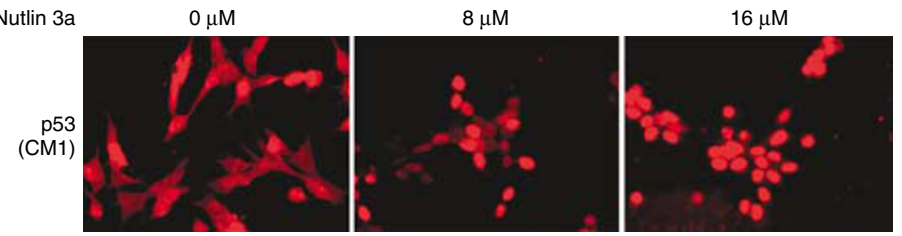

b

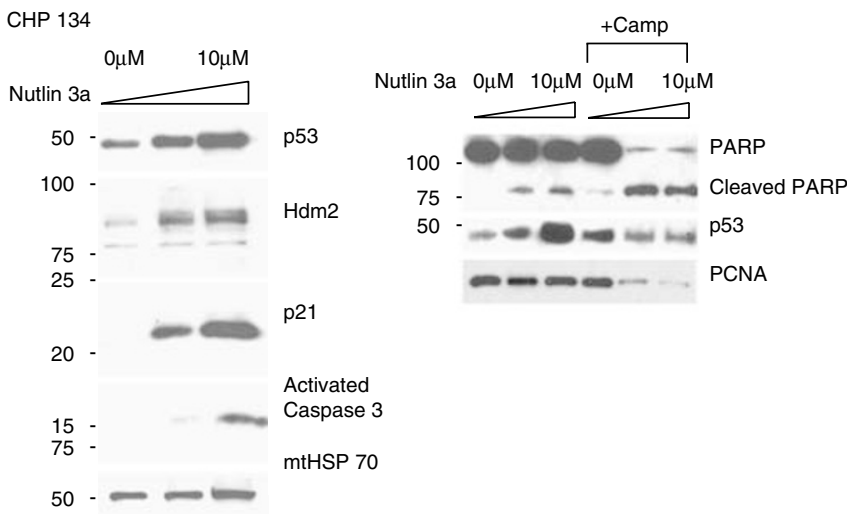

\begin{tabular}{l} 
CHP 134 \\
Nutlin $3 a$ \\
\hline
\end{tabular}

$50-\longrightarrow p 5$

100

utlin 3a ouM 10uM OuM $10 \mu \mathrm{M}$

DAPI
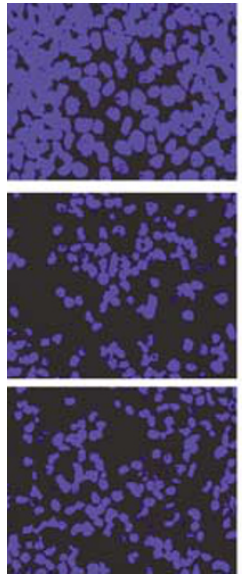

DAPI+TUNNEL
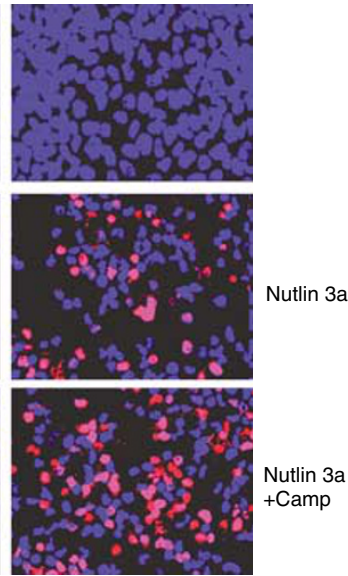

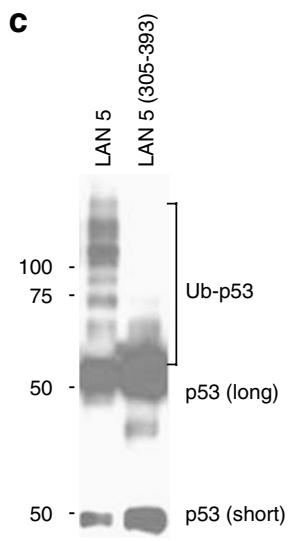

LAN 5

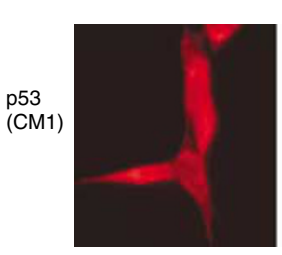

LAN 5 (305-393)

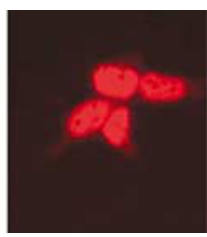

న్

மे

n 10 o $\quad$ IP:Hdm2

学忌 음

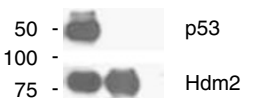


Immunoblotting and immunoprecipitation. For p53 ubiquitylation analysis, medium was removed and cells were quickly scraped and directly lysed by boiling in $100 \mu \mathrm{l}$ of reducing sample buffer $(0.1 \% \beta$-mercaptoethanol, $1 \mathrm{M}$ Tris- $\mathrm{HCl}, \mathrm{pH} 6.8$, bromophenol blue, $0.1 \% \mathrm{SDS}, 50 \%$ glycerol), subjected to $7.5 \%$ SDS-PAGE and transferred to nitrocellulose membranes. Immunoblot analysis was performed as we previously described and developed with Super Signal West Pico or Femto ECL (Pierce). ${ }^{7}$ In normalized p53 blots, a first blot was run to adjust loading for equal amounts of non-ubiquitylated p53 in the definitive second blot. For p21 and activated caspase-3 blotting, samples were run on $12 \%$ SDS-PAGE gels. For immunoprecipitation, cells scraped from confluent P100 dishes were lysed in $1 \mathrm{ml}$ IP buffer $(0.5 \%$ Triton X-100 in phosphate-buffered saline, protease inhibitor cocktail (Roche) and $50 \mathrm{ng} / \mathrm{ml}$ ubiquitinaldehyde (Sigma)) and sonicated. One milligram of total protein was incubated for $5 \mathrm{~h}$ at $4^{\circ} \mathrm{C}$ with $1 \mu \mathrm{g}$ of respective antibody and protein A/G Agarose beads (Roche) or, a cocktail of p53-specific D01/PAb1801 antibodies conjugated to agarose beads. The beads were washed four times in buffer $(0.5 \%$ Triton X-100 in PBS) and proteins were solubilized by boiling in $30 \mu \mathrm{l}$ of sample buffer before electrophoresis. The following antibodies were used: monoclonal D01 (Santa Cruz BioTechnology) and polyclonal rabbit CM1 (Vector) for p53, Hdm2 (monoclonal SMP 14 Santa Cruz; polyclonal rabbit: gift from J Chen), HAUSP (Calbiochem), Ubiquitin (P4D1, Santa Cruz), p21 (Santa Cruz), caspase-3 (Asp175, Cell Signalling), CRM 1 (BD Transduction Labs), mtHSP70 (ABR), PARC (polyclonal, gift from Dr. Wei Gu, Columbia University), $\beta$-importin (ABR). PCNA (Santa Cruz) was used as loading control, Lamin A/C (Sc 346, Santa Cruz) or HDAC1 (ABR) as nuclear marker and HSP 90 (ABR) as cytoplasmic marker. Mitochondria were purified by sucrose density gradients. ${ }^{40}$ In parallel, aliquots of cells were processed for crude lysates.

Immunofluorescence. Cells were grown on eight-chamber polystyrene slides, fixed for $3 \mathrm{~min}$ in acetone-methanol and air-dried. After blocking in $10 \%$ normal goat serum for $1 \mathrm{~h}$, cells were incubated in primary antibody overnight at $4^{\circ} \mathrm{C}$. Staining was detected with FITC- or TRITC-labelled anti-mouse and TRITClabelled anti-rabbit secondary antibodies (Jackson). Cells were mounted with Antifade (Molecular Probes) and Immunomount (Thermo) and examined with a Zeiss Axioskop.
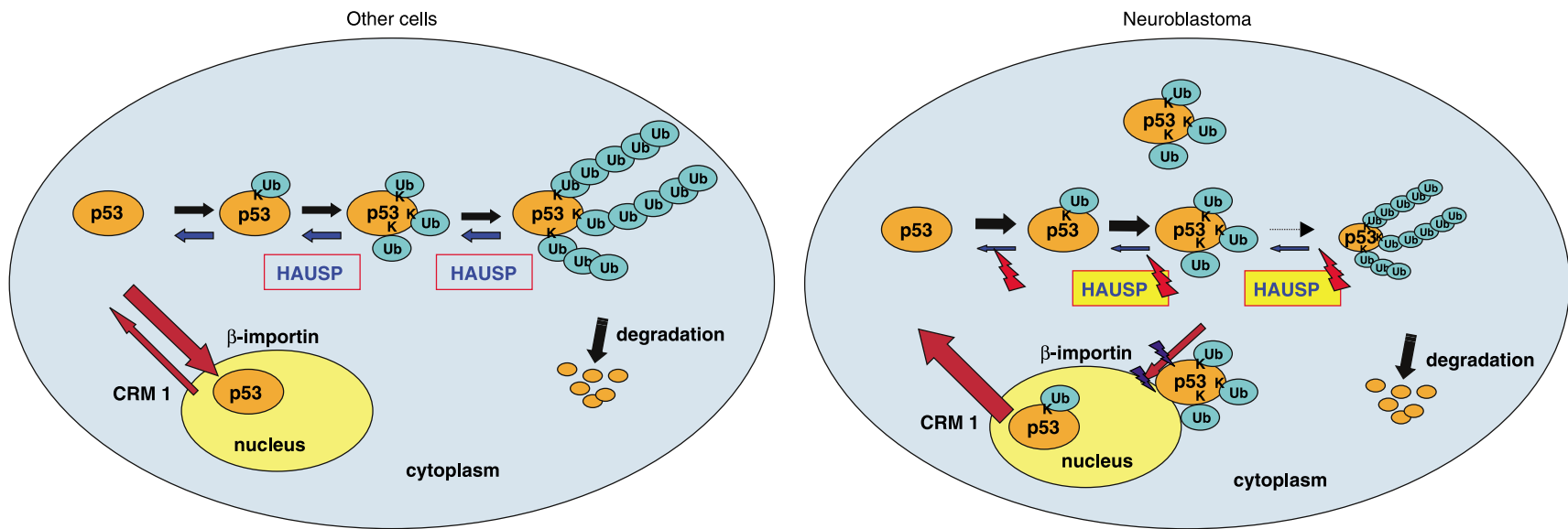

Figure 6 Model of p53 hyperubiquitylation in NB. Left. in normal cells p53 is avidly ubiquitylated on multiple C-terminal lysine residues by E3 ligases like Hdm2, and each of these ubiquitins serves as platform to build up ubiquitin chains. This polyubiquitylated p53 is rapidly degraded via the proteasome pathway. Of note, ubiquitylation is reversible by p53 deubiquitylating enzymes like HAUSP. Thus, HAUSP contributes to maintaining a stress-activatable p53 pool. Also p53, preferably the active non-ubiquitylated form, is imported into the nucleus, while p53 ubiquitylation is a prerequisite for its nuclear export. ${ }^{18-20}$ Right. in NB cells, the interaction between p53 and HAUSP is impaired, leading to constitutively hyperubiquitylated p53. As a result, the pool of non-ubiquitylated p53 decreases in favour of a gamut of ubiquitylated p53 species, of which only the polyUb forms are degradable while the (multi)monoUb species are stable and accumulate. As ubiquitylation of p53 favors its nuclear export, this shifts the balance of nucleo-cytoplasmic shuttling towards enhanced export, resulting in accumulation of hyperubiquitylated p53 in the cytoplasm. In addition, ubiquitylated p53 might also be impaired for nuclear import. For simplicity we only show cytoplasmic ubiquitylation of p53. However, we do not exclude the possibility that p53 is also ubiquitylated in the nucleus. Abbreviations: K, lysine residue, Ub, ubiquitin

Figure 5 Interference with $\mathrm{p} 53$ hyperubiquitylation in NB results in its relocalization from the cytoplasm to the nucleus and reactivation of p53 transcriptional and apoptotic function. (a) Left: Immunoblot of CHP134 NB cells treated with 0,8 and $16 \mu \mathrm{M}$ of the competitive Hdm2 inhibitor Nutlin $3 \mathrm{a}$ for $6 \mathrm{~h}$ followed by $2 \mathrm{~h}$ with ALLN for better visualization of ubiquitylated p53. Inhibition of the p53-Hdm2 interaction decreases the level of p53 ubiquitylation in a dose-dependent manner. Normalization for equal amounts of non-ubiquitylated p53. Right. Immunofluorescence of p53 (CM1) in CHP134 cells treated with increasing concentrations of Nutlin 3a. Increasing p53 deubiquitylation by Nutlin relocalizes p53 to the nucleus. While untreated cells exhibit moderate levels of diffuse cytoplasmic and nuclear p53 staining, rising Nutlin concentrations induce increasingly nuclear and finally exclusively nuclear p53 staining of higher intensity. (b) Nutlin-induced translocation of p53 to the nucleus is followed by reactivation of p53 function. Left. induction of p53 target genes Hdm2 and p21 and induction of apoptosis (activated caspase-3). Immunoblot of lysates from CHP134 cells treated with Nutlin3a $(0,5$ and $10 \mu \mathrm{M})$ for $6 \mathrm{~h}$. mtHSP 70 as loading control. Middle and right. Nutlin and camptothecin synergize to induce apoptosis in NB cells. Middle: CHP134 cells were seeded in 24-well plates and treated as indicated. Equal aliquots of crude lysates were immunoblotted for PARP and p53. PCNA is a loading control. The decrease in p53 and PCNA levels in lanes 5 and 6 is a reflection of massive apoptosis occurring under these conditions. Right. CHP134 cells were treated and stained for DAPI and TUNEL as indicated. (c) Top left. D01 immunoblot of LAN5 NB parental cells and a stable derivative line that expresses a C-terminal p53 fragment (aa 305-393), which lacks the N-terminal Hdm2-binding site and forms mixed heterotetramers with endogenous wtp53. p53 in these cells is stabilized and barely ubiquitylated, in contrast to parental LAN5. Top middle: Lack of p53 ubiquitylation in LAN5 (305-393) cells correlates with nuclear accumulation of p53, whereas hyperubiquitylated p53 in parental cells is significantly cytoplasmic. Immunofluorescence with CM1. Top right. Immunoprecipitation of p53, blotted for the nuclear export receptor CRM 1. Blot is normalized for equal amounts of p53. LAN5 cells have detectable interaction between p53 and CRM 1, whereas LAN5 (305-393) cells do not. The right lanes are direct immunoblots of input (equal protein). Bottom: immunoprecipitation of $\mathrm{Hdm} 2$ and blotting for $\mathrm{p} 53$ and $\mathrm{Hdm} 2$. Blots were normalized for equal amounts of precipitated $\mathrm{Hdm} 2$. Although endogenous wtp53 in LAN5 readily co-precipitates with Hdm2, the mixed wtp53/305-393 tetramers in LAN5(305-393) cells are completely unable to bind to Hdm2 
1. Tweddle DA, Pinkerton CR, Lewis IJ, Ellershaw C, Cole M, Pearson AD. OPEC/OJEC for stage 4 neuroblastoma in children over 1 year of age. Med Pediatr Oncol 2001; 36 239-242.

2. Vogelstein B, Lane D, Levine AJ. Surfing the p53 network. Nature 2000; 408: 307-310.

3. Keshelava N, Zuo JJ, Chen P, Waidyaratne SN, Luna MC, Gomer CJ et al. Loss of p53 function confers high-level multidrug resistance in neuroblastoma cell lines. Cancer Res 2001; 61: 6185-6193.

4. Tweddle DA, Malcolm AJ, Bown N, Pearson AD, Lunec J. Evidence for the development of p53 mutations after cytotoxic therapy in a neuroblastoma cell line. Cancer Res 2001; 61: 8-13.

5. Moll UM, LaQuaglia M, Benard J, Riou G. Wild-type p53 protein undergoes cytoplasmic sequestration in undifferentiated neuroblastomas but not in differentiated tumors. Proc Nat Acad Sci USA 1995; 92: 4407-4411.

6. Tweddle DA, Malcolm AJ, Cole M, Pearson AD, Lunec J. p53 cellular localization and function in neuroblastoma: evidence for defective $\mathrm{G}(1)$ arrest despite WAF1 induction in MYCN-amplified cells. Am J Pathol 2001; 158: 2067-2077.

7. Moll UM, Ostermeyer AG, Haladay R, Winkfield B, Frazier M, Zambetti G. Cytoplasmic sequestration of wild-type p53 protein impairs the $\mathrm{G} 1$ checkpoint after DNA damage. $\mathrm{Mol}$ Cell Biol 1996; 16: 1126-1137.

8. Nikolaev AY, Li M, Puskas N, Qin J, Gu W. Parc: a cytoplasmic anchor for p53. Cell 2003 112: $29-40$.

9. Stommel JM, Marchenko ND, Jimenez GS, Moll UM, Hope TJ, Wahl GM. A leucine-rich nuclear export signal in the p53 tetramerization domain: regulation of subcellular localization and p53 activity by NES masking. EMBO J 1999; 18: 1660-1672.

10. Zaika A, Marchenko N, Moll UM. Cytoplasmically 'sequestered' wild type p53 protein is resistant to Mdm2-mediated degradation. J Biol Chem 1999; 274: 27474-27480.

11. Ostermeyer AG, Runko E, Winkfield B, Ahn B, Moll UM. Cytoplasmically sequestered wildtype 553 protein in neuroblastoma is relocated to the nucleus by a $\mathrm{C}$-terminal peptide. Proc Natl Acad Sci USA 1996; 93: 15190-15194.

12. Rorie CJ, Weissman BE. The Ews/Fli-1 fusion gene changes the status of p53 in neuroblastoma tumor cell lines. Cancer Res 2004; 64: 7288-7295.

13. Wang $X$, Zalcenstein $A$, Oren $M$. Nitric oxide promotes $p 53$ nuclear retention and sensitizes neuroblastoma cells to apoptosis by ionizing radiation. Cell Death Differ 2003; 10 : 468-476.

14. Rodriguez-Lopez AM, Xenaki D, Eden TO, Hickman JA, Chresta CM. MDM2 mediated nuclear exclusion of p53 attenuates etoposide-induced apoptosis in neuroblastoma cells. Mol Pharmacol 2001; 59: 135-143.

15. Wolff A, Technau A, Ihling C, Technau-Ihling K, Erber R, Bosch FX et al. Evidence that wild-type p53 in neuroblastoma cells is in a conformation refractory to integration into the transcriptional complex. Oncogene 2001; 20: 1307-1317.

16. Nakamura S, Roth JA, Mukhopadhyay T. Multiple lysine mutations in the C-termina domain of p53 interfere with MDM2-dependent protein degradation and ubiquitination. $\mathrm{Mol}$ Cell Biol 2000; 20: 9391-9398.

17. Rodriguez MS, Desterro JM, Lain S, Lane DP, Hay RT. Multiple C-terminal lysine residues target $\mathrm{p} 53$ for ubiquitin-proteasome-mediated degradation. Mol Cell Biol 2000; 20: $8458-8467$.

18. Lohrum MA, Woods DB, Ludwig RL, Balint E, Vousden KH. C-terminal ubiquitination of $p 53$ contributes to nuclear export. Mol Cell Biol 2001; 21: 8521-8532.

19. Boyd SD, Tsai KY, Jacks T. An intact HDM2 RING-finger domain is required for nuclear exclusion of p53. Nat Cell Biol 2000; 2: 563-568.
20. Geyer RK, Yu ZK, Maki CG. The MDM2 RING-finger domain is required to promote p53 nuclear export. Nat Cell Biol 2000; 2: 569-573.

21. Li M, Brooks CL, Wu-Baer F, Chen D, Baer R, Gu W. Mono- versus polyubiquitination: differential control of p53 fate by Mdm2. Science 2003; 302: 1972-1975.

22. Li M, Chen D, Shiloh A, Luo J, Nikolaev AY, Qin J et al. Deubiquitination of p53 by HAUSP is an important pathway for p53 stabilization. Nature 2002; 416: 648-653.

23. Cummins JM, Rago C, Kohli M, Kinzler KW, Lengauer C, Vogelstein B. Tumour suppression: disruption of HAUSP gene stabilizes p53. Nature 2004; 428: 1 p following 486.

24. Li M, Brooks CL, Kon N, Gu W. A dynamic role of HAUSP in the p53-Mdm2 pathway. Mol Cell 2004; 13: 879-886.

25. Meulmeester E, Maurice MM, Boutell C, Teunisse AF, Ovaa H, Abraham TE et al. Loss of HAUSP-mediated deubiquitination contributes to DNA damage-induced destabilization of Hdmx and Hdm2. Mol Cell 2005; 18: 565-576.

26. Isaacs JS, Hardman R, Carman TA, Barrett JC, Weissman BE. Differential subcellular p53 localization and function in N- and S-type neuroblastoma cell lines. Cell Growth Differ 1998 9: 545-555.

27. Appella $E$, Anderson CW. Post-translational modifications and activation of p53 by genotoxic stresses. Eur J Biochem 2001; 268: 2764-2772.

28. Stommel JM, Wahl GM. Accelerated MDM2 auto-degradation induced by DNA-damage kinases is required for p53 activation. EMBO J 2004; 23: 1547-1556.

29. Moll UM, Marchenko N, Zhang XK. p53 and Nur77/TR3 - transcription factors that directly target mitochondria for cell death induction. Oncogene 2006; 25: 4725-4743.

30. Carr J, Bell E, Pearson AD, Kees UR, Beris $\mathrm{H}$, Lunec $\mathrm{J}$ et al. Increased frequency of aberrations in the p53/MDM2/p14(ARF) pathway in neuroblastoma cell lines established at relapse. Cancer Res 2006; 66: 2138-2145.

31. Vassilev LT, Vu BT, Graves B, Carvajal D, Podlaski F, Filipovic $Z$ et al. In vivo activation of the p53 pathway by small-molecule antagonists of MDM2. Science 2004; 303: 844-848.

32. Shirangi TR, Zaika A, Moll UM. Nuclear degradation of $p 53$ occurs during down-regulation of the p53 response after DNA damage. FASEB J 2002; 16: 420-422.

33. Thrower JS, Hoffman L, Rechsteiner M, Pickart CM. Recognition of the polyubiquitin proteolytic signal. EMBO J 2000; 19: 94-102.

34. Gu J, Nie L, Wiederschain D, Yuan ZM. Identification of p53 sequence elements that are required for MDM2-mediated nuclear export. Mol Cell Biol 2001; 21: 8533-8546.

35. Hu M, Gu L, Li M, Jeffrey PD, Gu W, Shi Y. Structural basis of competitive recognition of p53 and MDM2 by HAUSP/USP7: implications for the regulation of the p53-MDM2 pathway. PLoS Biol 2006; 4: e27.

36. Marchenko ND, Wolff S, Erster S, Becker K, Moll UM. Monoubiquitylation promotes mitochondrial p53 translocation. EMBO J 2007 (E-pub ahead of print).

37. Masuya D, Huang C, Liu D, Nakashima T, Yokomise H, Ueno M et al. The HAUSP gene plays an important role in non-small cell lung carcinogenesis through p53-dependent pathways. J Pathol 2006; 208: 724-732.

38. Barbieri E, Mehta P, Chen Z, Zhang L, Slack A, Berg S et al. MDM2 inhibition sensitizes neuroblastoma to chemotherapy-induced apoptotic cell death. Mol Cancer Ther 2006; 5 2358-2365.

39. Van Maerken T, Speleman F, Vermeulen J, Lambertz I, De Clercq S, De Smet E et al. Small-molecule MDM2 antagonists as a new therapy concept for neuroblastoma. Cancer Res 2006; 66: 9646-9655.

40. Marchenko ND, Zaika A, Moll UM. Death signal-induced localization of p53 protein to mitochondria. A potential role in apoptotic signaling. J Biol Chem 2000; 275: 16202-16212.

Supplementary Information accompanies the paper on Cell Death and Differentiation website (http://www.nature.com/cdd) 